\title{
Monitoring and analysis of sea fog in an offshore waterway using lidar
}

\author{
Lijun Hu$\odot^{\text {a,b,c,* } * \text { and Hao Yang }}{ }^{\mathrm{a}}$ \\ ${ }^{a}$ Ningbo Meteorological Network and Equipment Support Center, Ningbo, China \\ ${ }^{b}$ Zhejiang Meteorological Information and Network Center, Hangzhou, China \\ ${ }^{\mathrm{c} C h i n a}$ Meteorological Administration, Key Laboratory of Atmosphere Sounding, \\ Chengdu, China
}

\begin{abstract}
Until now, sea fog monitoring relied mainly on point monitoring equipment, such as a forward scatter visibility sensor mounted on the shore or on an island, which produce non-ideal data during a variable and unevenly distributed sea fog. In 2019, a visibility lidar instrument for sea fog monitoring was installed in the Beilun area of the Ningbo Zhoushan Port, China. Two sea fog events were monitored in February 2020, and the data from the lidar were compared with those from a forward scatter visibility sensor. The results show that the visibility lidar instrument is advantageous for sea fog monitoring, and the correlation of the lidar instrument data with the forward scatter sensor data demonstrates the utility and potential of the lidar for sea fog detection. (1) The Authors. Published by SPIE under a Creative Commons Attribution 4.0 Unported License. Distribution or reproduction of this work in whole or in part requires full attribution of the original publication, including its DOI. [DOI: 10.1117/1.OE.60.6.064103]
\end{abstract}

Keywords: Beilun port; waterway sea fog; visibility lidar; forward scatter visibility sensor.

Paper 20210305 received Mar. 25, 2021; accepted for publication Jun. 2, 2021; published online Jun. 18, 2021.

\section{Introduction}

Fog is characterized by the suspension of very small droplets and ice crystals of water in air that results in a visibility of $<1 \mathrm{~km}\left(5 / 8\right.$ of a statute mile). ${ }^{1,2}$ Fog and its effect on visibility (Vis) have significant impacts on our daily lives. ${ }^{3}$ In navigation, sea fog is a disastrous weather event that substantially affects the safety of ships. Losses related to fog and low visibility have become comparable to those caused by other weather events, such as tornadoes or hurricanes. ${ }^{2}$ Generally, the thickness of sea fog ranges from 200 to $400 \mathrm{~m}$; however, it can sometimes be over $\sim 500 \mathrm{~m}^{4}$ The various fog types include advection fog, mixed fog, radiation fog, and terrain fog. The horizontal range and vertical height of the fog change widely and rapidly. Accurate forecasts could help to mitigate the financial losses and reduce injuries caused by fog. ${ }^{5}$ However, accurate fog forecasting/nowcasting is difficult because of the inherent difficulties in detecting fog. ${ }^{2}$ Over the years, the meteorological service in the wharf of the Ningbo Zhoushan Port in China has depended on a few scatter-type visibility sensors to acquire sea fog and associated data. During a systematic sea fog occurrence (i.e., a wide range of fog within a weather system, such as advection fog), adequately referenced monitoring data from a forward scatter visibility sensor $^{6,7}$ can represent the surrounding area. However, because of the complexity of sea channel fog, the forward scatter sensor exhibits limitations in monitoring, making it impossible for the sensor to provide early warning observation under highly variable sea fog conditions. Conversely, as lidar is characterized by good monochromaticity, strong directionality, and high coherence, it has been widely employed in environmental and meteorological monitoring as well as other fields. Lidar-based observations are typically used to obtain Doppler wind speed. Lidar can also provide horizontal wind profiles to analyze turbulence and gust at low elevation angles. Recently, lidar has been used for aviation research and operations, including marine fog studies. ${ }^{8,9}$

The Ningbo Zhoushan Port in China comprises 19 areas, including Beilun, Yangshan, Liuheng, Qushan, and Chuanshan. In 2020, the cargo throughput in this port was estimated

*Address all correspondence to Lijun Hu, qxthlj@sina.com 
at 1.172 billion tons, ranking it the first in the world for the 12th consecutive year. Consequently, the meteorological fine (i.e., comprehensive and targeted) service demands, including the routing of ships, wharf stop, and port area operation, are very high, especially when sea fog ${ }^{10-12}$ is present. Therefore, to improve the meteorological services, the Ningbo Meteorological Department built a modern marine monitoring system in the port. In 2018, a visibility lidar instrument was used for a sea fog monitoring test in the Beilun area of the Ningbo Port by the Ningbo Meteorological Bureau and Darsun Laser Technology Co., Ltd. based on the conventional lidar technology for cloud height and shape determination. After almost a year of testing, comparison, and improvement, the lidar monitoring was finalized. In the present study, the monitoring data and performance of the visibility lidar instrument are compared to those acquired by forward scatter visibility sensors during two sea fog events that occurred between February 11 and February 16, 2020.

\section{Lidar Principle}

As the laser emitted by the lidar system is transmitted to the atmosphere, different atmospheric components absorb and scatter the laser, ${ }^{13,14}$ thereby altering the energy, spectral characteristics, and polarization state of the backscattered light. Based on the optical properties of the backscattered light, the corresponding visibility information can be retrieved. ${ }^{15,16}$ Therefore, the visibility lidar instrument is considered a backscattered light receiver capable of receiving laser echo signals from tens of thousands of meters away. Through computer analysis, the atmospheric extinction coefficient and associated parameters, as well as visibility data, are obtained.

The measurement principle of backscattering visibility lidar is that the signal $P(r)$ of the laser that is backscattered by the atmosphere, with a central position $r$ and thickness $\Delta r$, can be expressed as follows: ${ }^{13,17}$

$$
P(r)=T_{L} \mathrm{E}_{0} \mathrm{cA}_{\mathrm{R}}(r) \frac{A}{r^{2}} \Delta r \beta(r) \tau^{2}(r)+B,
$$

where $E_{0}(J), T_{L}$, and $A_{R}$ denote the single laser pulse energy, total transmittance of the emission system, and effective area of the receiving telescope $\left(\mathrm{m}^{2}\right)$, respectively; $c$ denotes the speed of light $(\mathrm{m} / \mathrm{s})$, and $\tau(r)=\exp \left(-\int_{0}^{r} \alpha\left(r^{\prime}\right) \mathrm{d} r^{\prime}\right)$ is the one-way atmospheric transmittance factor. The parameters $\alpha$ is the extinction coefficient, whereas $\beta$ is the atmospheric backscattering coefficient. Parameter $B$ includes the background noise associated with all electronics and optics. The extinction and backscattering coefficients comprise two components, namely contributions from molecules and aerosols, expressed as follows: ${ }^{13,17}$

$$
\left\{\begin{array}{l}
\alpha=\alpha_{a e r}+\alpha_{m o l} \\
\beta=\beta_{a e r}+\beta_{m o l}
\end{array}\right.
$$

where the subscripts aer and $\mathrm{mol}$ represent the contributions from aerosols and molecules, respectively. In the lower boundary layer of the visibility lidar system, the contribution from aerosols is higher than that from molecules; therefore, Eq. (2) can be simplified as follows:

$$
\left\{\begin{array}{l}
\alpha \approx \alpha_{\text {aer }} \\
\beta \approx \beta_{\text {aer }}
\end{array}\right.
$$

The extinction coefficient $\alpha$ is extracted using the horizontal visibility inversion algorithm, ${ }^{18}$ which overcomes the sensitivity of the initial value without requiring the lidar ratio (i.e., extinction to backscattering coefficients ratio). The $V_{\mathrm{MOR}}$ is defined as follows: ${ }^{19}$

$$
V_{\mathrm{MOR}}=-\frac{1}{\alpha} \ln K^{\prime}=\frac{3.912}{\alpha},
$$

where $K^{\prime}$ is the visual contrast threshold of the human eye.

If the laser wavelength used differs from $550 \mathrm{~nm}$, then the atmospheric extinction coefficient $\alpha(r)$, detected by the lidar instrument, requires wavelength modification. The empirical correction formula is as follows: ${ }^{20,21}$ 


$$
V_{\mathrm{MOR}}=\frac{3.912}{\alpha}\left(\frac{\lambda}{0.55}\right)^{-q}
$$

The coefficient $q$ is determined using the following empirical formula: ${ }^{20,21}$

$$
q= \begin{cases}0.585 V^{\frac{1}{3}} & V<6 \mathrm{~km} \\ 1.3 & 6 \mathrm{~km} \leq V<50 \mathrm{~km} \\ 1.6 & V \geq 50 \mathrm{~km}\end{cases}
$$

\section{Observation and Project Site}

The offshore channel of the Beilun area of the Ningbo Zhoushan Port is the main route for international containers and large cargo ships. Owing to the presence of many islands in the channel, sea fog significantly obstructs the navigation of vessels. Forward scatter visibility sensors and a visibility lidar instrument are installed near the channel; the locations of those used in the present study are shown in Fig. 1.

Under the same weather conditions, a larger wavelength corresponds to a smaller extinction coefficient and subsequent lower signal attenuation, resulting in a longer detection distance. However, in terms of signal-to-noise ratio, Ref. 18 reports that a shorter wavelength is more favorable for a shorter detection range. Therefore, a visibility lidar with a wavelength of $532 \mathrm{~nm}$ was selected for the analysis and was installed in the Beilun area of the Ningbo to monitor sea fog in the offshore waterway. The horizontal scanning visibility lidar instrument (Model: DSL-V021-3D) manufactured by Darsun Laser Technology Co., Ltd. China can effectively acquire visibility distribution information along a path by detecting the backscattering of the laser interaction with different atmospheric components. Thus, the point monitoring limitation associated with a forward scatter visibility sensor can be compensated. The main parameters of the visibility lidar instrument in the Beilun area are presented in Table 1.

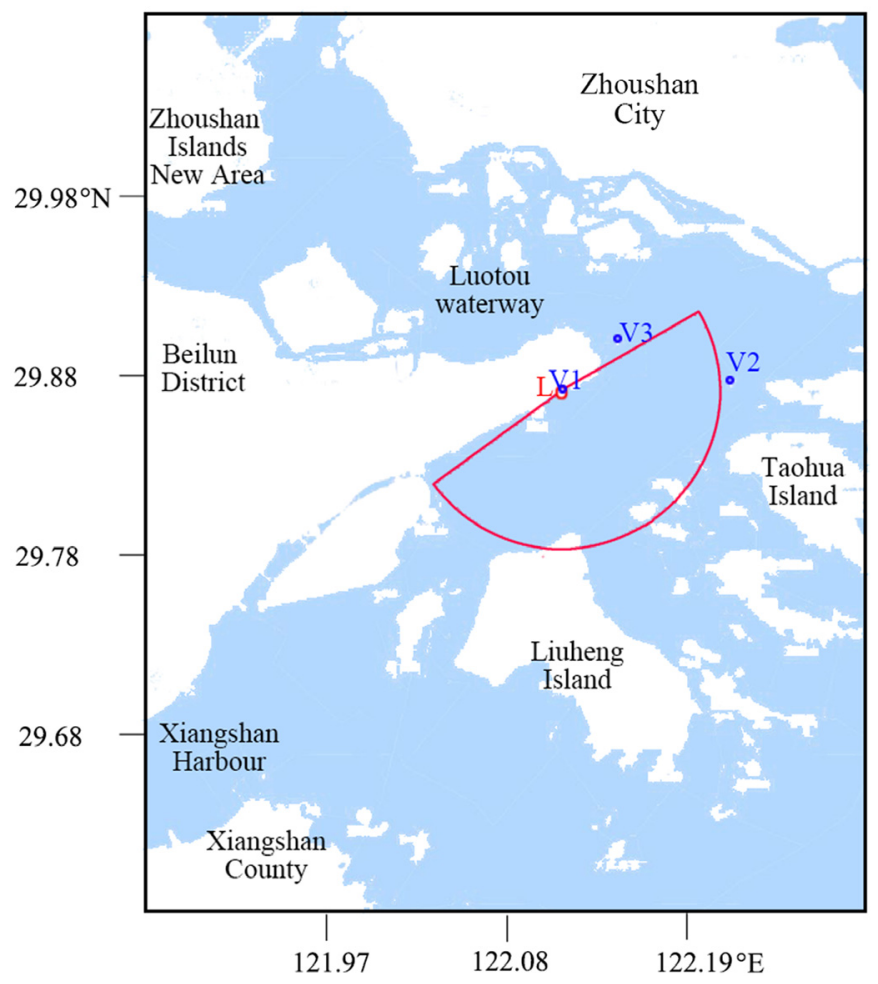

Fig. 1 Map of the Ningbo Zhoushan Port area showing the locations of the visibility lidar and forward scatter visibility sensors. 
Table 1 Summary of data parameters used in assessing the performance of the visibility lidar instrument.

\begin{tabular}{lcccccc}
\hline \hline & & Single & Spatial & & \\
Model & Laser source & pulse energy & resolution & Time resolution & Frequency & Pulse width \\
\hline $\begin{array}{l}\text { DSL-V021-3D/DSL- } \\
\text { V022-3D }\end{array}$ & $532 \mathrm{~nm}$ & $100 \mu \mathrm{J}$ & $15 \mathrm{~m}$ & $5 \mathrm{~s}$ to $10 \mathrm{~min}$ & 1 to $10 \mathrm{kHz}$ & $<10 \mathrm{~ns}$ \\
\hline \hline
\end{tabular}

Table 2 Forward scatter visibility measurement specifications.

\begin{tabular}{lcccc}
\hline \hline Model & Measurement range & Accuracy & Time constant & Update interval \\
\hline GJY-1G(J) & $10 \mathrm{~m}$ to $30 \mathrm{~km}$ & $\begin{array}{l}+/-10 \%, \text { range } 10 \text { to } 10 \mathrm{~km} \\
+/-20 \%, \text { range } 10 \text { to } 30 \mathrm{~km}\end{array}$ & $60 \mathrm{~s}$ & $15 \mathrm{~s}$ \\
\hline \hline
\end{tabular}

The scanning azimuth of the visibility lidar instrument shown in Fig. 1 varies from 60 deg to $232 \mathrm{deg}$; the scanning angle resolution is $2 \mathrm{deg}$, and the measurement time is $10 \mathrm{~s}$. Therefore, the lidar instrument's average scan cycle time is $\sim 15 \mathrm{~min}$. The scanned echo signal, characterized by a semicircular shape centered around the location of the lidar instrument, is processed using an inversion algorithm ${ }^{22,23}$ to produce a visibility map.

The notations V1, V2, and V3, indicated by blue dots in Fig. 1, represent three forward scatter visibility sensors [Model: GJY-1G(J)] manufactured by Airborne Missile Academy Environment Monitoring Co., Ltd. China). Table 2 lists the main parameters of the forward scatter visibility in the Beilun area. The Symbol L, marked by the red circle, represents a visibility lidar instrument mounted at an 8-m altitude site, with a wavelength of $532 \mathrm{~nm}$. The sector represents the monitoring area associated with the lidar instrument. The comparison of the monitoring data of the visibility lidar and the three forward scatter visibility sensors revealed that valid data were missing for the V1 meter because of its location in the blind spot of the lidar monitoring instrument. The V3 meter is near the monitoring range of the lidar instrument ( $\sim 4.5 \mathrm{~km}$ distance at an angle of $50 \mathrm{deg}$ from the lidar instrument installation point), whereas the V2 meter is $10.9 \mathrm{~km}$ from the visibility lidar station. Therefore, if the laser beam scans at 0.5 deg elevation and 2 deg resolution, the height is $95 \mathrm{~m}$ at $10.9 \mathrm{~km}$, with a sector area involving a monitoring range of $35 \times 15 \mathrm{~m}^{2}$. The geographic locations of the forward scatter visibility sensors at the surface may vary, causing differences in the data acquired, especially during uneven weather conditions. Therefore, the data from the V3 meter were selected for comparison with the data from the visibility lidar instrument. The site of the V3 meter is at an altitude of $50 \mathrm{~m}$, and the scan height of the laser is $47 \mathrm{~m}$ near the V 3 meter. The visibility detected using the visibility lidar at $\mathrm{V} 3$ is used to compare with those detected using forward scatter visibility sensors at $\mathrm{V} 1$ and $\mathrm{V} 3$.

\section{Results and Discussion}

Multiple low-visibility events occurred between February 11 and February 13, 2020, as shown in Fig. 2. In operation, the forward scatter visibility sensor takes the scattering coefficient as the approximate substitute for the extinction coefficient and ignores the absorption coefficient. Consequently, when the visibility is high, the error is relatively large. ${ }^{6}$ In contrast, the visibility lidar considers both the scattering coefficient and the absorption coefficient; thus, its result is more accurate than that of the forward scatter visibility sensor. In this paper, we focus on lowvisibility conditions such as sea fog. Therefore, to eliminate the influence of large error of forward scattering visibility in high-visibility weather, forward scattering visibility and lidar visibility above $20 \mathrm{~km}$ are unified as $20 \mathrm{~km}$ in Figs. 2 and 4. At 0:00 on February 11, the visibility range is $\sim 8 \mathrm{~km}$ [Fig. 3(a)], which decreases to below $1 \mathrm{~km}(958 \mathrm{~m})$ at 08:32, during the onset of fog. The visibility range then increases or decreases with the change of sea fog, and subsequently increases rapidly after 12:00 on February 12 due to sea fog dissipation. Initially, visibility starts 


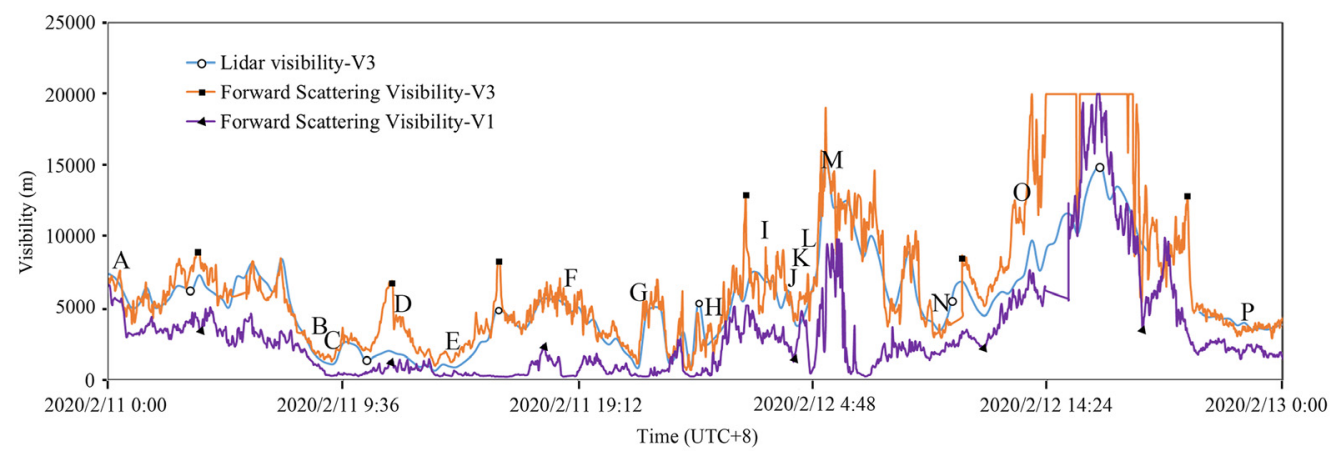

Fig. 2 Comparison of the lidar visibility-V3 and forward scattering visibility-V1 and V3 for the period February 11-13, 2020.

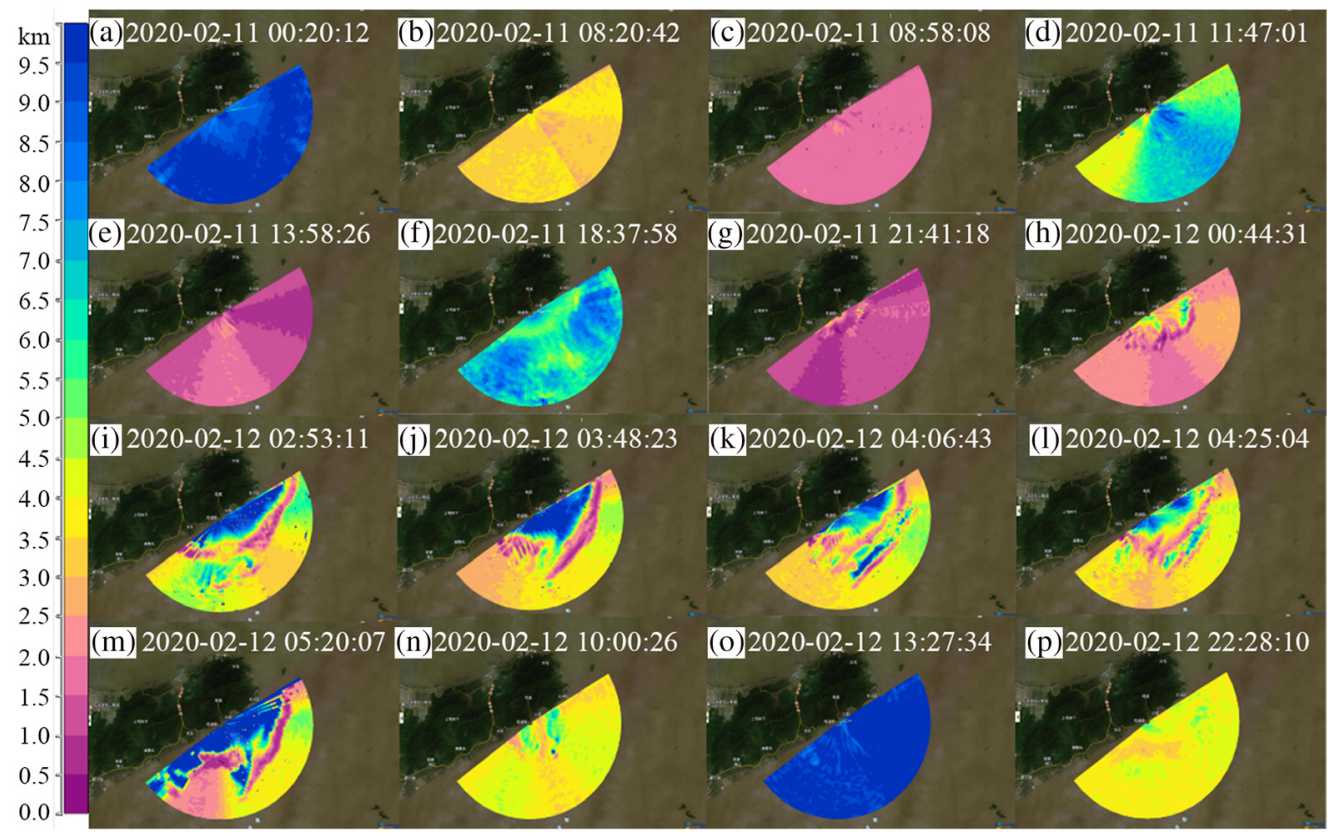

Fig. 3 Scan images from the visibility lidar instrument at different times (UTC+8) during February 11-13, 2020. A-P correspond to the times at A-P in Fig. 2.

declining at 08:00 on February 11, as shown in Fig. 2, and the corresponding visibility lidar instrument image at this point is shown in Fig. 3(b). Visibility declines to $\sim 1.4 \mathrm{~km}$ by around 09:00, as shown in Fig. 3(c), before improving to $\sim 6 \mathrm{~km}$ at around 12:00, as shown in Fig. 3(d). Thereafter, visibility begins to decline again, reaching $\sim 1.2 \mathrm{~km}$ at 14:00, as shown in Fig. 3(e), and then increases to $6.2 \mathrm{~km}$ at around 18:00, as shown in Fig. 3(f). The images in Figs. 3(h)-3(m) reflect the sea fog changes on the sea surface between 0:00 and 05:00 on February 12. Evidently, visibility is high in the central paths of the lidar and forward scatter visibility sensors, although some sea fog persists on the sea. During this period, the variation trend of the visibility data obtained by V3 is nearly equal to that obtained by lidar, demonstrating the reliability of the lidar monitoring data. Simultaneously, the lidar map can clearly reflect the movement of small-scale fog, from a few hundred meters to a few kilometers, between 00:00 and 06:00 on February 12, while V1 and V3 cannot represent the fog situation on the sea surface, indicating that lidar has certain advantages in monitoring sea surface visibility. After 06:00, the fog begins shifting toward the central paths, causing the visibility range to decline to $\sim 4 \mathrm{~km}$, before it starts rising at around 14:00, reaching $\sim 20 \mathrm{~km}$. However, after 18:00, the visibility range starts to decline again. This indicates that the density of sea fog varies considerably with 


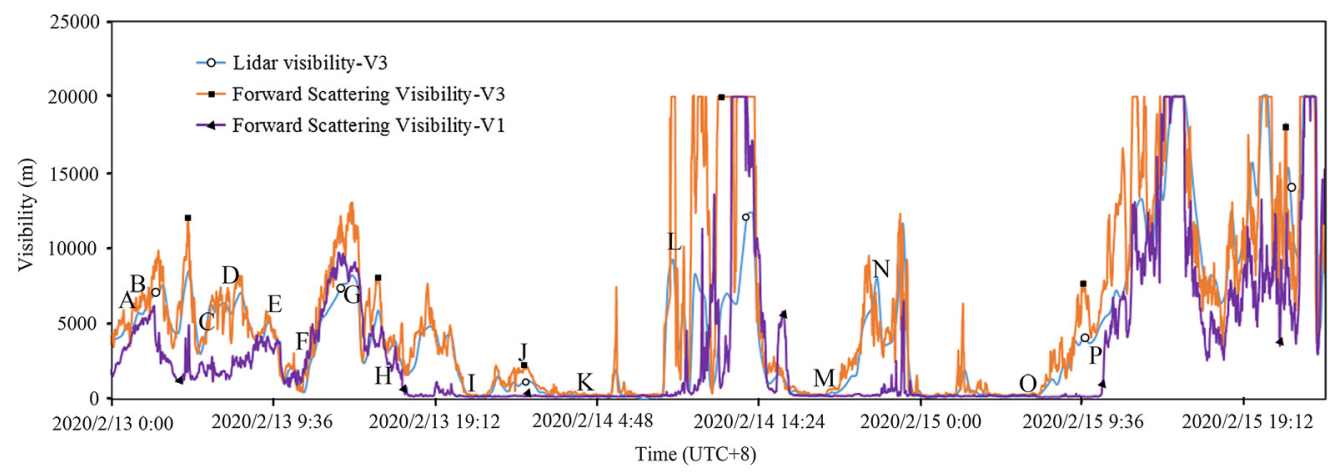

Fig. 4 Comparison of the lidar visibility-V3 and forward scattering visibility-V1 and V3 for the period February 13-16, 2020.

time and space and that lidar can well reflect the changes of sea fog, especially when this occurs in patches (i.e., duration in minutes). ${ }^{1}$

Several low-visibility events also occurred during February 13-16, 2020, as shown in Fig. 4. On February 13, the visibility range is $\sim 4 \mathrm{~km}$ because of the presence of sea fog, as shown in Fig. 5(a). The images in Figs. 5(a)-5(e) reflect the variations in the visibility range above the sea from 0:00 to 09:30. Figure 4 shows that the coast visibility obtained by V1 is lower than that of the sea surface represented by V3 simultaneously. However, there is no evidence of small-scale sea fog on the sea surface in the scattered distribution of forward scatter visibility. At around 11:00, the visibility range decreases to $\sim 600 \mathrm{~m}$ due to light or moderate fog above the sea, as displayed in Figs. 4(f) and 5(f), before rising to $\sim 10 \mathrm{~km}$ at 14:30 [Fig. 5(g)] and then decreasing to $\sim 3 \mathrm{~km}$ at around 16:00 after sea fog re-emerges [Fig. 5(h)]. The visibility variations between 21:00 on February 13 and 03:00 on February 14 are shown in Figs. 5(i)-5(k). The visibility range during this period is generally $<1 \mathrm{~km}$. At around 09:00 on February 14, the V3 visibility range attained $20 \mathrm{~km}$, despite the presence of sea fog, as exhibited in Fig. 5(1). These findings again indicate the inhomogeneity of sea fog. At around 18:00, the site was again covered by sea fog, as shown in Fig. 5(m), until dissipation occurred at around 21:00, as shown in Fig. 5(n). After 23:00, the visibility started declining, maintaining ranges $<1 \mathrm{~km}$ from 0:00 to 10:00 on

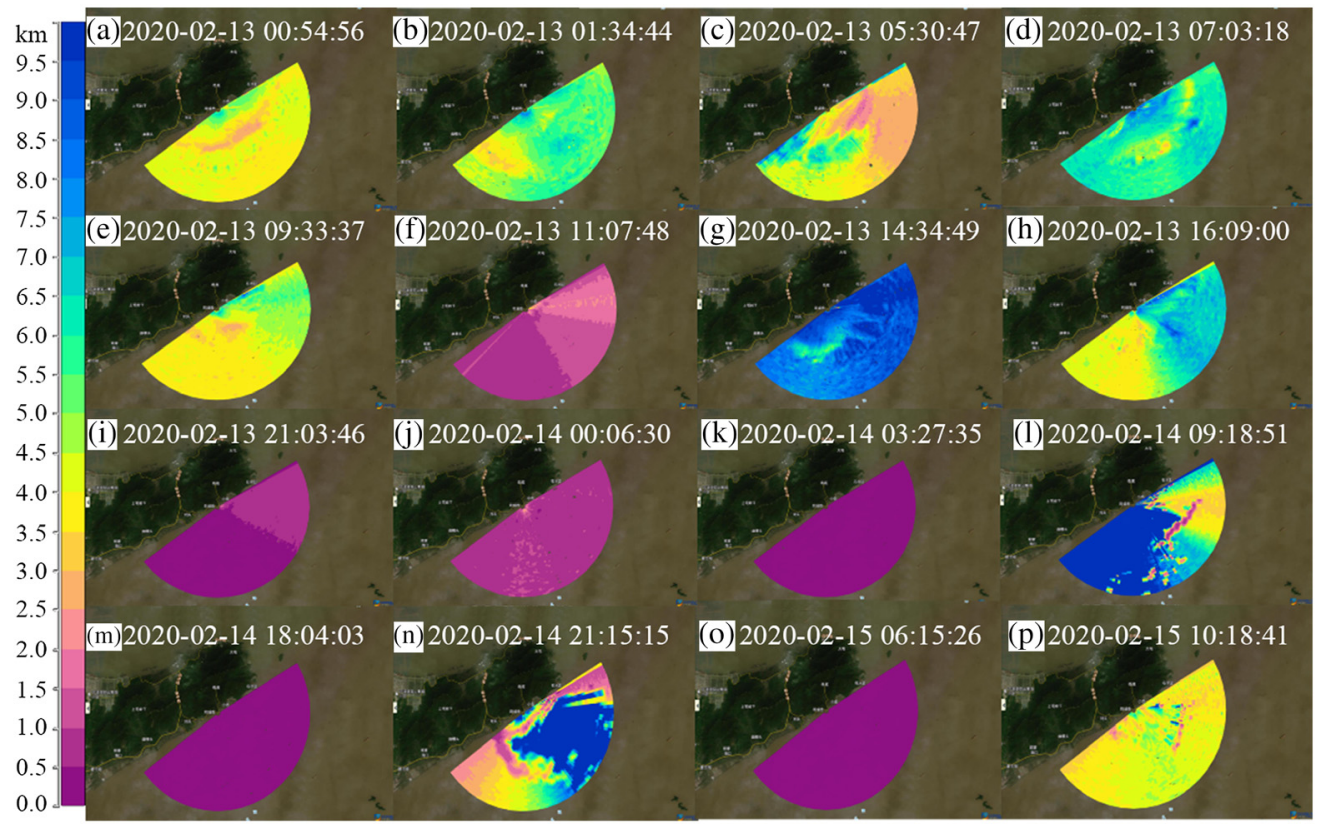

Fig. 5 Visibility lidar images associated with different times (UTC+8) during February 13-16, 2020. A-P correspond to the times at A-P in Fig. 4. 


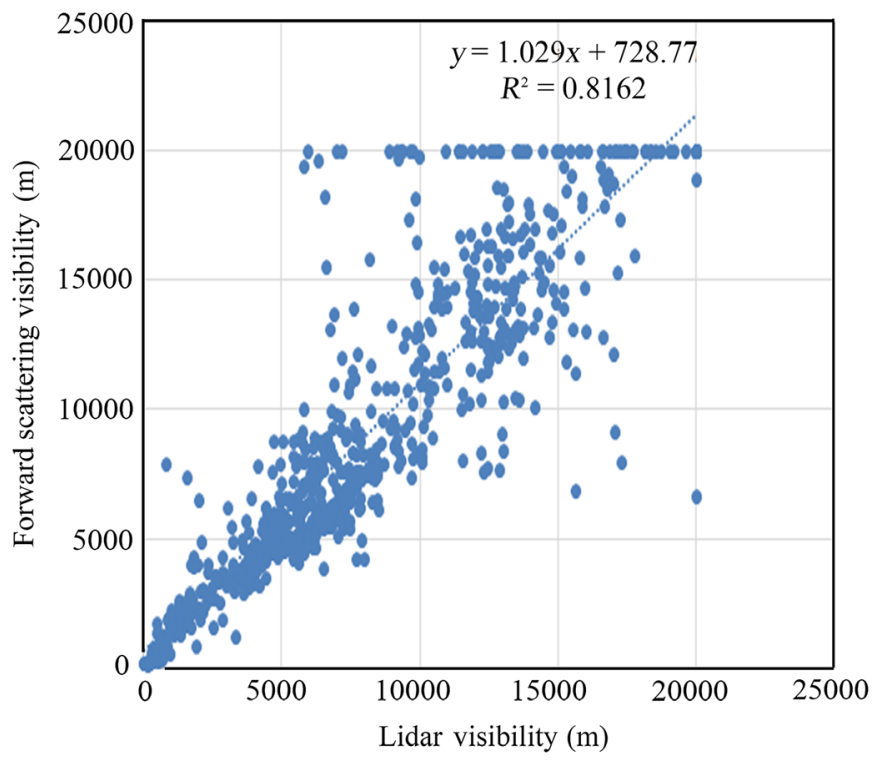

Fig. 6 Plot of the visibility data from the forward scatter sensor versus the laser visibility instrument showing the correlation coefficient.

February 15, as shown in Fig. 5(o). After 10:00, the visibility range improves, reaching $\sim 5 \mathrm{~km}$, as shown in Fig. 5(p). Figures 5(k)-5(p) show that there are several occurrences of moderate to thick fog between 03:00 on February 14 and 10:00 on the 15th, with each fog event lasting several hours. During the large periods of thick fog, the visibility of V1 and V3 monitoring are consistent with that of lidar monitoring, and both can represent the visibility on the nearby sea surface. Lidar maps presented in Figs. 5(1) and 5(n) indicate that the visibility on the sea surface considerably differ sometimes. The sea fog showed an intermittent long strip movement, thereby fully reflecting the advantages of visibility lidar. Therefore, the visibility lidar can obtain the details of sea fog change and movement for any duration.

The relationship between the data from the forward scatter visibility sensor and the visibility lidar instrument under different visibility conditions is shown in Fig. 6. The two methods exhibit a strong positive correlation $\left(R^{2}>0.8\right)$, thereby supporting the suitability of the lidar system for visibility monitoring in the port. When the actual visibility exceeds $10 \mathrm{~km}$, the average absolute deviation is $1890 \mathrm{~m}$; when it is $<5 \mathrm{~km}$, the average absolute deviation is $314 \mathrm{~m}$.

\section{Conclusions}

Unlike the forward scatter visibility sensor, the visibility lidar technology involves the detection and analysis of light backscattering caused by the interaction between atmospheric particles and the laser emitted from the instrument. The technology exhibited suitability for characterizing uneven atmospheric visibility, thereby effectively providing section-by-section information on visibility distribution in an entire detection path. In the present study, the visibility lidar technology is used to monitor sea fog and fog agglomeration in sea areas, including ports. The following conclusions are drawn from this work:

1. The comparison of data from multiple low-visibility events that occurred between February 11 and 16, 2020 in the offshore channel of the Beilun Port indicates that the forward scatter visibility sensor on the shore cannot detect adequately the visibility and fog on the sea.

2. The inhomogeneity of sea fog distribution varies considerably with time and space in inshore or offshore channels.

3. Lidar monitoring of fog over the sea is more reliable and superior than the nearby forward scatter sensor for large area fog monitoring. 
4. The visibility lidar map can clearly reflect the movement of small-scale fog, which demonstrates certain advantages of lidar compared to the forward scatter sensor.

5. Lidar can accurately reflect the changes and variations in sea fog, particularly marine fog occurring in patches or exhibiting a long strip movement.

The results show that lidar can serve in offshore channel visibility monitoring of the port area. In future research, several visibility lidars will be used to form a monitoring network suitable for visibility detection over a wide area.

\section{Disclosures}

The authors declare no conflict of interest.

\section{Acknowledgments}

The authors are thankful to Darsun Laser Technology Co., Ltd. China for the assistance provided. This research was supported by the Zhejiang Provincial Natural Science Foundation of China (Grant No. LGF21D050003) and Key Laboratory for Atmospheric Sounding of China (Grant No. 2021KLAS02Z), Ningbo Meteorological Science and Technology Plan Project (Grant No. NBQXTD2019002).

\section{References}

1. S. G. Kutty et al., "Fog occurrence and associated meteorological factors over Kempegowda International Airport, India," J. Pure Appl. Geophys. 176, 2179-2190 (2019).

2. I. Gultepe et al., "Fog research: a review of past achievements and future perspectives," J. Pure Appl. Geophys. 164, 1121-1159 (2007).

3. I. Gultepe et al., "The fog remote sensing and modeling (FRAM) field project: visibility analysis and remote sensing of fog," Proc. SPIE 7088(12), 708-803 (2008).

4. L. Hu et al., "Application of millimeter wave radar in sea fog monitoring in Beilun port area," Meteorol. Sci. Technol. 48, 171-177 (2020).

5. I. Gultepe et al., "Introduction ice fog, ice clouds, and remote sensing," J. Pure Appl. Geophys. 173, 2977-2982 (2016).

6. J. V. Winstanley and M. J. Adams, "Point visibility meter - Forward scatter instrument for measurement of aerosol extinction coefficient," Appl. Opt. 14(9), 2151-2157 (1975).

7. R. Barrales-Guadarrama et al., "A new forward-scatter visibility sensor based on a universal frequency-to-digital converter," Instrum. Sci. Technol. 41(5), 445-462 (2013).

8. I. Gultepe et al., "A review of high impact weather for aviation meteorology," J. Pure Appl. Geophys. 176, 1869-1921 (2019).

9. I. Gultepe et al., "Marine fog: a review on microphysics and visibility prediction," in Marine Fog: Challenges and Advancements in Observations, Modeling, and Forecasting, D. Koračin and C. E. Dorman, Eds., pp. 345-394, Springer, Switzerland (2017).

10. D. Koračin et al., "Marine fog: a review," Atmos. Res. 143, 142-175 (2014).

11. J. Lewis et al., "Sea fog off the California coast: viewed in the context of transient weather systems," J. Geophys. Res. 108(D15), 4457 (2003).

12. J. Chen et al., "Analysis of a sea fog episode at King George Island, Antarctica," Atmosphere 10(10), 585 (2019).

13. F. G. Fernald, B. M. Herman, and J. A. Reagan, "Determination of aerosol height distributions by lidar," J. Appl. Meteorol. 11(3), 482-489 (1972).

14. J. D. Spinhirne, "Micro pulse lidar," IEEE Trans. Geosci. Remote Sens. 31(1), 48-55 (1993).

15. M. Chen et al., "Detective method and analysis of atmospheric slant visibility for lidar," Infrared Laser Eng. 35, 156-160 (2006).

16. F. Castagnoli et al., "Lidar system for visibility monitoring," Opt. Quantum Electron. 15(3), 261-268 (1983). 
17. R. M. Measures, Laser Remote Sensing: Fundamentals and Applications, Krieger, Florida (1992).

18. J. Xian et al., "Parameter optimization of a visibility LiDAR for sea-fog early warnings," Opt. Express. 28, 23829-23845 (2020).

19. J. L. Miller et al., Photonics Rules of Thumb, 3rd ed., SPIE Press, Bellingham, Washington (2020).

20. W. Paul et al., Elements of Infrared Technology, Generation, Transmission, and Detection, Wiley, New York (1962).

21. A. Ångström, "The parameters of atmospheric turbidity," Tellus 16, 64-75 (1964).

22. J. Xian et al., "Novel Lidar algorithm for horizontal visibility measurement and sea fog monitoring," Opt. Express 26(26), 34853-34863 (2018).

23. A. Pantazis, A. Papayannis, and G. Georgousis, "Lidar algorithms for atmospheric slant range visibility, meteorological conditions detection, and atmospheric layering measurements," Appl. Opt. 56(23), 6440-6449 (2017).

Lijun Hu is a senior engineer at the Ningbo Meteorological Network and Equipment Support Center. He received his bachelor's degree in atmospheric exploration from Chengdu Meteorological Institute in 1995 and a master's degree in Information and Communication Engineering from the University of Science and Technology of China (USTC) in 2007. He has authored more than 20 journal articles. His current research interests include sea fog research using lidar and information technology approaches. He is a member of the China Computer Federation.

Hao Yang is an engineer at the Ningbo Meteorological Network and Equipment Support Center. $\mathrm{He}$ received his bachelor's degree in communications engineering from Xi' an University of Science and Technology in 2010 and master's degree in computer from the University of Ningbo in 2019. Currently, he is engaged in meteorological equipment support. He has written or participated in the writing of more than 10 journal papers. His research interests are computer technology applications and atmospheric sounding. 